\title{
Clinical Differences between Men and Women with Psoriatic Arthritis: Relevance of the Analysis of Genes and Polymorphisms in the Major Histocompatibility Complex Region and of the Age at Onset of Psoriasis
}

\author{
Rubén Queiro,, ${ }^{1,2}$ Patricia Tejón,, ${ }^{1}$ Pablo Coto, ${ }^{2,3}$ Sara Alonso, ${ }^{1}$ \\ Mercedes Alperi, ${ }^{1}$ Cristina Sarasqueta, ${ }^{4}$ Segundo González, ${ }^{5}$ Jesús Martínez-Borra, ${ }^{6}$ \\ Carlos López-Larrea, ${ }^{6}$ and Javier Ballina ${ }^{1}$ \\ ${ }^{1}$ Rheumatology Department, Hospital Universitario Central de Asturias (HUCA), C/Celestino Villamil s/n, \\ Oviedo, 33006 Asturias, Spain \\ ${ }^{2}$ Psoriasis Unit, Hospital Universitario Central de Asturias (HUCA), Oviedo, Asturias, Spain \\ ${ }^{3}$ Dermatology Department, Hospital Universitario Central de Asturias (HUCA), Oviedo, Asturias, Spain \\ ${ }^{4}$ Clinical Epidemiology Unit, BIODONOSTIA, San Sebastian, Basque Country, Spain \\ ${ }^{5}$ Department of Functional Biology, Oviedo University School of Medicine, Oviedo, Asturias, Spain \\ ${ }^{6}$ Immunology Service, HUCA, Oviedo, Asturias, Spain \\ Correspondence should be addressed to Rubén Queiro; rubenque7@yahoo.es
}

Received 16 September 2012; Revised 17 March 2013; Accepted 28 March 2013

Academic Editor: Chung Tei Chou

Copyright (C) 2013 Rubén Queiro et al. This is an open access article distributed under the Creative Commons Attribution License, which permits unrestricted use, distribution, and reproduction in any medium, provided the original work is properly cited.

\begin{abstract}
It has been shown that males with spondyloarthritis tend to suffer from more severe spinal disease while females are more likely to have peripheral joint involvement. Nevertheless, gender-related differences have not been thoroughly explored in psoriatic arthritis (PsA). In PsA, males accumulate more peripheral and axial joint damage compared to women. However, it is not clear whether these findings are secondary to differences in occupational physical activity, hormonal changes, or other factors. The present study analyzed the differences in clinical expression of PsA between men and women. We have also evaluated the possible existence of gender-linked differences in the distribution of genes and polymorphisms within the major histocompatibility complex and whether patients' age at the onset of psoriasis established any differences in these aspects. Women suffered more polyarthritis, greater functional impairment, and a larger number of swollen joints during followup. We appreciated a differential expression of certain MHC genes according to gender and age at onset of psoriasis. Our results point to the need to include patient's age at the onset of psoriasis and gender as key stratification elements in future studies of genetic associations in PsA.
\end{abstract}

\section{Introduction}

Psoriasis is a relatively common T-cell mediated cutaneous disease in which both environmental and genetic factors contribute to its pathogenesis [1]. Despite multiple susceptibility loci for psoriasis that have been identified, the PSORS1 locus located in the MHC class I region on chromosome 6 p21.3 confers the most risk for psoriasis [1]. However, the extensive ranges of polymorphism and the preservation of HLA haplotypes in most populations have made it difficult to characterize the exact gene responsible for psoriasis susceptibility located in PSORS1. Nevertheless, HLA-C ${ }^{*} 06$ is the strongest association found in psoriasis and is the only genetic variant repeatedly observed to be associated with psoriasis in most of the populations (1). Additionally, other MHC genes have also been associated with psoriasis independently of HLA-C* $06[2,3]$.

Psoriatic arthritis (PsA) is a chronic inflammatory joint disease which affects a significant proportion (10-40\%) of psoriatic patients $[4,5]$. HLA-C ${ }^{*} 06$ has also been associated with PsA susceptibility (6). MICA-A9 (corresponding to MICA $\left.^{*} 002\right)$ polymorphism has been further associated 
with arthritis susceptibility but not with psoriasis [6-8]. Additionally, HLA-B38, HLA-B39, HLA-B57, HLA-DR7, and TNF- $\alpha$ promoter polymorphisms have been associated with PsA susceptibility in the past; however, these alleles are in linkage disequilibrium (LD) with HLA-C ${ }^{*} 06$ or MICAA9 [6-8]. Thus, it has been postulated that there are two different susceptibility loci associated with PsA in the MHC region: HLA-C ${ }^{*} 06$, which is associated with the skin lesions (present in the extended haplotypes-EH-13.1, 37.1, and 57.1) and another, MICA-A9, associated with susceptibility to arthritis and present in EH38.1, EH39.1, and EH57.1. Therefore, haplotype EH57.1 (HLA-C* 06-B57-DRB1* 07-DQA1*02$\left.\mathrm{DQB1}^{*} 03\right)$ is associated with both psoriasis and inflammatory arthropathy.

It has been shown that males with spondyloarthritis tend to suffer from more severe spinal disease while females are more likely to have peripheral joint involvement [9]. Nevertheless, gender-related differences have not been thoroughly explored in PsA. In a recent investigation among patients with PsA, men tended to accumulate more peripheral and axial joint damage compared to women. However, it was unclear whether these findings were secondary to differences in occupational physical activity, hormonal changes, or other factors [10]. It is also plausible that some of these genderobserved differences in PsA could be attributed to genetic factors. Indeed, some studies have shown a close correlation between male sex, HLA-B27 positivity, and risk of psoriatic spondyloarthritis $[11,12]$. Very few studies have investigated whether there are differences in the clinical and genetic risk profiles between men and women with PsA [13].

The present study analyzes the differences in clinical expression of PsA between men and women. We have also evaluated the possible existence of gender-linked differences in the distribution of genes and polymorphisms within the MHC and whether patients' age at the onset of psoriasis establishes any differences in these aspects.

\section{Patients and Methods}

2.1. Patients. One-hundred ten consecutive patients who fulfilled the Classification criteria for psoriatic arthritis (CASPAR) criteria [14] were randomly selected from the rheumatology outpatient clinic of a tertiary care hospital. There were 55 men and 55 women with a mean age of $48 \pm 12$ years. The mean disease duration for psoriasis was $19 \pm 10$ years and $13 \pm 8$ years for arthritis. Psoriasis preceded the arthritis in $75 \%$ of cases and a family history of psoriasis was recorded in $43 \%$ of patients. The joint patterns on the baseline visits were those described by Moll and Wright [15], though in the final analysis increased emphasis was placed on the dominant joint pattern over the last 5 years of followup. Accordingly, $39 \%$ had oligoarthritis, $29 \%$ polyarthritis, and 32\% presented a predominantly axial disease. Distal interphalangeal joint disease was seen in $32 \%$ of patients, whereas nail disease was seen in $43.6 \%$. Dactylitis was noted in $30 \%$ of patients. Clinical enthesitis was present in $30 \%$ of subjects. All the disease variables, including laboratory, clinical and exploratory tests, disease activity, physical function, joint counts, and radiological studies were investigated on a standard basis in both men and women. The population was stratified by age at the time of onset of psoriasis, adopting a cutoff point of 40 years for distinguishing between early and late psoriasis. The study was carried out with the approval of the ethics committee of our hospital. All patients gave written informed consent before enrolling in the study.

2.2. HLA Typing. Human leukocyte antigen, HLA-C, was typed using the polymerase chain reaction with sequencespecific primers (PCR-SSP) [7]. The polymorphisms of the octamer transcription factor 3 gene (OTF3), the corneodesmosin gene (CDSN), and the $a$-helix coiled-coil rod homologue (HCR) gene were analyzed as described previously [7]. The microsatellites $\mathrm{C}_{\_} \_25$ and $\mathrm{C}_{1} \_4 \_4$ were amplified using the PCR primers described by Tamiya et al. [16]. HLA-B typing was carried out using the Dynal RELI SSO HLA-B test following the manufacturer's instructions. HLA-DRB1 alleles were typed and subtyped by performing polymerase chain reaction using specific primers (PCR-SSP). For the analysis of microsatellite repeat polymorphism in the MICA gene, PCR was carried out using primers labeled at the $5^{\prime}$ end with the fluorescent reagent $\mathrm{Cy} 5$ as previously described [7]. For MICB typing, a dinucleotide microsatellite polymorphism in the first intron was analyzed by PCR analysis and TNF- $\alpha$ polymorphism at positions -238 and -308 was typed by PCR as previously described [7]. The distribution of these markers was analyzed in the whole group as well as in both men and women separately. This distribution was also analyzed according to patients' age at the onset of psoriasis, using the previously mentioned cutoff point. All previous typing was also performed in a control population of 110 (55 men and 55 women) random blood donors matched ethnically and geographically.

2.3. Statistical Analysis. The differences between the frequencies of these allelic markers in patients and controls, as well as the differences found in accordance with sex and age at disease onset, were assessed by univariate analysis using a Fisher's exact test, chi-squared test with Yates' correction and a Student's $t$-test. The differences between genders corresponding to the categorical variables were assessed with Fisher's exact test, while Student's $t$-test was used in the case of the continuous variables. The odds ratio (OR) was calculated by the cross-product ratio and the $95 \%$ confidence intervals by the cornfield method. The $P$ significant values were corrected $\left(P_{c}\right)$ by multiplying them by the number of alleles at each locus. The extent of linkage disequilibrium between two loci is expressed as the observed disequilibrium value $\left(\lambda_{s}\right)$, that is, a proportion of the theoretical maximum disequilibrium value $\left(\lambda_{\max }\right)$ achievable for this combination of alleles. The $\lambda_{\mathrm{s}}$ were calculated using the formula: $\lambda_{\mathrm{s}}=$ $\lambda / \lambda_{\text {max }}=\mathrm{Pab}-(\mathrm{Pa} \cdot \mathrm{Pb}) / \mathrm{Pa} \cdot(1-\mathrm{Pb})$.

\section{Result}

3.1. Differences in the Clinical Variables of Disease between Men and Women. Compared to PsA males, females tended 
TABLE 1: Clinical characteristics of the study population.

\begin{tabular}{|c|c|c|c|}
\hline Variables & $\begin{array}{l}\text { Men } \\
n: 55\end{array}$ & $\begin{array}{c}\text { Women } \\
n: 55\end{array}$ & $\begin{array}{c}P \\
\text { values }\end{array}$ \\
\hline Age (yr) & $46.5 \pm 11.6$ & $46.4 \pm 15.9$ & NS \\
\hline Psoriasis onset age (yr) & $27.5 \pm 10.4$ & $27.0 \pm 14.9$ & NS \\
\hline Arthritis onset age (yr) & $34.2 \pm 9.4$ & $35.1 \pm 13.1$ & NS \\
\hline Psoriasis duration (yr) & $19 \pm 11$ & $18.2 \pm 8.8$ & NS \\
\hline Arthritis duration (yr) & $13 \pm 7.4$ & $12.5 \pm 6.7$ & NS \\
\hline Psoriasis-arthritis latency (yr) & $7 \pm 6.4$ & $7.5 \pm 5.6$ & NS \\
\hline Family history & $40 \%$ & $45.5 \%$ & NS \\
\hline Oligoarthritis & $42 \%$ & $34.5 \%$ & NS \\
\hline Polyarthritis & $20 \%$ & $40 \%$ & $<0.05$ \\
\hline Axial disease & $38.2 \%$ & $23.6 \%$ & NS \\
\hline Nail disease & $36.4 \%$ & $47.3 \%$ & NS \\
\hline DIP disease & $29 \%$ & $34.5 \%$ & NS \\
\hline IBP (ever) & $62 \%$ & $67.3 \%$ & NS \\
\hline Enthesitis & $34.5 \%$ & $25.5 \%$ & NS \\
\hline Dactylitis & $31 \%$ & $31 \%$ & NS \\
\hline HAQ & $0.62 \pm 0.42$ & $1.10 \pm 0.53$ & $<0.01$ \\
\hline Swelling joint count & $3.4 \pm 4.2$ & $5.2 \pm 4.6$ & $<0.05$ \\
\hline Tender joint count & $10.2 \pm 3.4$ & $11.0 \pm 4.1$ & NS \\
\hline Erosive disease & $34.5 \%$ & $41.8 \%$ & NS \\
\hline PASI & $5.4 \pm 4.5$ & $5.7 \pm 3.8$ & NS \\
\hline VSG & $15 \pm 5.3$ & $16 \pm 4.7$ & NS \\
\hline HLA-C ${ }^{*} 06$ & $56.4 \%$ & $56.4 \%$ & NS \\
\hline HLA-B27 & $36.4 \%$ & $27.3 \%$ & NS \\
\hline
\end{tabular}

DIP: distal interphalangeal joint. IBP: inflammatory back pain. HAQ: Health Assessment Questionnaire. PASI: Psoriasis Area and Severity Index.

to be affected more frequently by polyarthritis as the main joint pattern during followup ( $40 \%$ versus $20 \%, P<0.05$ ), higher HAQ values $(1.10 \pm 0.53$ versus $0.62 \pm 0.42, P<$ $0.01)$, and higher swelling joint count $(5.2 \pm 4.6$ versus 3.2 $\pm 4.2 P<0.05)$. There were no gender differences in age at the onset of psoriasis or arthritis, family history of disease, distal interphalangeal involvement, dactylitis, nail disease, the presence of erosive disease in the radiological study, or severity of psoriasis (Table 1).

\subsection{Differences in Disease Variables between Men and Women} according to Age at the Onset of Psoriasis (Cutoff Point 40 Years). Most patients developed psoriasis before 40 years of age (45 men and 40 women). These individuals showed a family history of psoriasis in 47 cases (40\%), while the rest of the patients with psoriasis first manifesting after 40 years of age presented antecedents of the disease in only three cases (12\%), $P<0.05$. In general, the psoriasis-arthritis latency period was significantly shorter in patients with late psoriasis $(3.5 \pm 3.4$ years) than in subjects with early psoriasis $(7.3 \pm 5.4$ years) $(P<0.01)$. The only discordant data between genders in the patients with psoriasis developing before 40 years of age were a shorter psoriasis-arthritis latency period in men $(5.5 \pm 7.1$ years $)$ than in women $(9.3 \pm 6.6$ years $)(P<0.01)$, and a greater frequency of polyarthritis during followup in women $(37.5 \%$ versus $17.7 \%)(P<0.05)$. Women with early psoriasis showed greater nail involvement than men, though the difference was not statistically significant. In women with type II psoriasis, polyarthritis likewise was the dominant PsA pattern (53.3\% versus 30\%), though the differences failed to reach statistical significance, due to the small number of cases in this group. In the same way, in men with type II psoriasis, the dominant joint pattern was axial involvement $(70 \%$ versus $33.3 \%)$, though once again the differences failed to reach statistical significance, due to the small number of cases in this group (Table 2).

3.3. Comparative Distribution of MHC Genetic Markers between Men and Women. The global study population showed a relationship between the presence of HLA-C ${ }^{*} 06$ (56.4\% versus $17 \%$, OR 6.18, 95\% CI: $3.32-11.5, P_{c}<0.0001$ ), MICA-A9 (60\% versus 30\%, OR 3.5, 95\% CI: 2.0-6.12, $P_{c}<$ 0.001 ), and HLA-B 27 (31.8\% versus $7.3 \%$. OR 5.9, 95\% CI: 2.6-13.4, $\left.P_{c}=0.001\right)$ and the risk of suffering PsA. Subjects expressing HLA-C ${ }^{*} 06$ were significantly younger at the time of onset of psoriasis $(24 \pm 13.6$ years) than the individuals without this allele $(32.4 \pm 14.1$ years $), P=0.01$. With respect to HLA-C ${ }^{*} 06$ and family history, $64 \%$ of subjects who showed a positive family history had this allele compared to $44 \%$ of those with no family history, OR $2.3,95 \%$ CI: $0.82-$ $6.36, P=0.08$. MICA-A9 showed no association with a family history of psoriasis or with patient's age at onset of the disease. However, the patients with allele 384 of the $\mathrm{Cl}_{-} 44_{-}$ microsatellite, telomeric to HLA-C, were significantly older at the onset of psoriasis $(35.3 \pm 12.6$ versus $25.1 \pm 13.7$ years, $P=0.01)$ and of arthritis ( $42 \pm 11.5$ versus $33 \pm 11.7$ years, $P=0.01)$. In the same way as MICA-A9, this microsatellite was not associated with a family history of disease.

The following markers were overexpressed in women with PsA: HLA-B ${ }^{*} 27$ (27.3\% versus $7.3 \%$. OR 4.8, 95\% CI: $\left.1.5-15.5, P_{c}=0.01\right)$, HLA-C 06 (56.4\% versus $16.4 \%$. OR 6.6, $95 \%$ CI: $\left.2.7-16.1, P_{c}=0.0001\right), C^{*} 07$ (49\% versus $25.5 \%$. OR 2.8, 95\% CI: $\left.1.3-6.3, P_{c}=0.01\right)$, TNF-308A (45.5\% versus $22 \%$. OR 3.0, 95\% CI: 1.3-6.9, $\left.P_{c}=0.009\right)$, and MICA-A9 (60\% versus $32.7 \%$. OR 3.1, 95\% CI: $1.4-6.7, P_{c}=0.004$ ). In turn, in men only the following markers were significantly elevated with respect to the male control population: HLA$\mathrm{C}^{*} 06$ (56.4\% versus $18.2 \%$. OR 5.8, 95\% CI: $2.4-13.8, P_{c}=$ $0.0001)$, HLA-B ${ }^{*} 27$ (36.4\% versus $7.3 \%$. OR 7.3, 95\% CI: $2.3-$ $\left.23.2, P_{c}=0.0004\right)$, and MICA-A9 (60\% versus $27.3 \%$. OR 4.0 , $95 \%$ CI: $1.8-8.9, P_{c}=0.001$ ).

In reference to the relationship between the genes regulating disease risk and the differential clinical features, 17 out of 24 women with nail disease were $C^{*} 06$ positive (71\%) versus only 6 out of 20 males with nail disease (30\%), $P<0.01$. The psoriasis-arthritis latency period was significantly shorter in MICA-A9 positive men ( $5.4 \pm 6.6$ years) than in women with this marker $(8.2 \pm 6.4$ years), $P<0.01$. Similarly, the psoriasisarthritis latency period was significantly shorter in men with the 384 allele of C4_1_1 (5.1 \pm 6.1 years) than in women with this same microsatellite ( $8.1 \pm 6.1$ years), $P<0.01$. 
TABLE 2: Differences between men and women according to age at psoriasis onset (cutoff: 40 years).

(a) Age at psoriasis onset $<40$

\begin{tabular}{lccc}
\hline Variable & Men & Women & $P$ \\
& $n: 45$ & $n: 40$ & values \\
\hline Age (yr) & $46.3 \pm 8.5$ & $48.6 \pm 14.6$ & NS \\
Arthritis onset age (yr) & $35.5 \pm 6.8$ & $37.6 \pm 10.6$ & NS \\
Psoriasis onset age (yr) & $25 \pm 8.2$ & $22 \pm 9.9$ & NS \\
Psoriasis duration (yr) & $16.3 \pm 14.1$ & $21 \pm 9.2$ & 0.07 \\
Arthritis duration (yr) & $10.9 \pm 9.5$ & $11 \pm 8.9$ & NS \\
Psoriasis-arthritis latency (yr) & $5.5 \pm 7.1$ & $9.3 \pm 6.6$ & $<0.01$ \\
Family history & $44.4 \%$ & $50 \%$ & NS \\
Oligoarthritis & $46.7 \%$ & $40 \%$ & NS \\
Polyarthritis & $17.7 \%$ & $37.5 \%$ & $<0.05$ \\
Axial disease & $33.3 \%$ & $22.5 \%$ & NS \\
DIP disease & $26.7 \%$ & $40 \%$ & NS \\
Nail disease & $37.8 \%$ & $52.5 \%$ & NS \\
Enthesitis & $35.6 \%$ & $27.5 \%$ & NS \\
Dactylitis & $25.5 \%$ & $35 \%$ & NS \\
Erosive disease & $33.3 \%$ & $40 \%$ & NS \\
HAQ & $0.7 \pm 4.5$ & $0.8 \pm 6.2$ & NS \\
SJC & $3.3 \pm 4.6$ & $4.3 \pm 3.2$ & NS \\
TJC & $8.2 \pm .5 .2$ & $9.2 \pm 5.5$ & NS \\
PASI & $7.3 \pm 3.4$ & $8.1 \pm 4.4$ & NS \\
\hline & & & \\
& $5.3 \%$ & & \\
& & &
\end{tabular}

(b) Age at psoriasis onset $>40$

\begin{tabular}{|c|c|c|c|}
\hline Variable & $\begin{array}{l}\text { Men } \\
n: 10\end{array}$ & $\begin{array}{c}\text { Women } \\
n: 15\end{array}$ & $\begin{array}{c}P \\
\text { values }\end{array}$ \\
\hline Age (yr) & $59.4 \pm 8.4$ & $67.1 \pm 8.2$ & 0.03 \\
\hline Arthritis onset age (yr) & $49.4 \pm 3.4$ & $52.5 \pm 9.6$ & NS \\
\hline Psoriasis onset age (yr) & $45.7 \pm 3.4$ & $51.2 \pm 6.9$ & $<0.05$ \\
\hline Psoriasis duration (yr) & $13.6 \pm 8$ & $15.9 \pm 5.3$ & NS \\
\hline Arthritis duration (yr) & $9.9 \pm 5.9$ & $14.5 \pm 9$ & NS \\
\hline Psoriasis-arthritis latency (yr) & $3.7 \pm 3.3$ & $3.2 \pm 3.6$ & NS \\
\hline Family history & $10 \%$ & $13.3 \%$ & NS \\
\hline Oligoarthritis & $0 \%$ & $20 \%$ & NS \\
\hline Polyarthritis & $30 \%$ & $53.3 \%$ & NS \\
\hline Axial disease & $70 \%$ & $33.3 \%$ & NS \\
\hline DIP disease & $30 \%$ & $13.3 \%$ & NS \\
\hline Nail disease & $30 \%$ & $20 \%$ & NS \\
\hline Enthesitis & $30 \%$ & $20 \%$ & NS \\
\hline Dactylitis & $30 \%$ & $20 \%$ & NS \\
\hline Erosive disease & $40 \%$ & $46.7 \%$ & NS \\
\hline HAQ & $0.8 \pm 5.1$ & $0.9 \pm 5.3$ & NS \\
\hline SJC & $3.2 \pm 4.6$ & $4.7 \pm 4.4$ & NS \\
\hline TJC & $10.3 \pm 4.1$ & $11.3 \pm 5.2$ & NS \\
\hline PASI & $4.2 \pm 2.3$ & $3.8 \pm 3.6$ & NS \\
\hline
\end{tabular}

DIP: distal interphalangeal joint. HAQ: Health Assessment Questionnaire. SJC: swelling joint count. TJC: tender joint count. PASI: Psoriasis Area and Severity Index.
3.4. Distribution of Genetic Markers according to Patient Age at Onset of Psoriasis. There were no significant differences in age at the onset of psoriasis between men and women with early psoriasis and HLA-C 06 positivity $(21 \pm 8.3$ versus $18.1 \pm 8.9$ years, resp.). However, among the individuals with late psoriasis (over 40 years of age) and HLA-C* 06 positivity, the age at onset of psoriasis was significantly younger in men ( $44.3 \pm 3.2$ years) than in women ( $57 \pm 4.2$ years), $P<0.01$. Similarly, men with C1_4_4 (384) microsatellite positivity and the development of psoriasis after 40 years of age were significantly younger at disease onset $(45.7 \pm 3.8$ years $)$ than the women with the same marker (53.3 \pm 7 years), $P<0.01$. The women developing psoriasis before 40 years of age ( $n$ $=40$ ) showed significant elevation of the following markers versus the female control population: HLA-C ${ }^{*} 06$ (65\% versus 16.4\%. OR 9.5, 95\% CI: 3.6-24.9, $\left.P_{c}=0.0001\right)$, HLA-C 07 (50\% versus $25.5 \%$. OR 2.9, 95\% CI: $1.2-6.9, P_{c}=0.02$ ), HLA${ }^{*} \mathrm{~B} 27$ (30\% versus 7.3\%. OR 5.5, 95\% CI: $\left.1.6-18.5, P_{c}=0.005\right)$, and MICA-A9 (62.5\% versus $32.7 \%$. OR 3.4, 95\% CI: $1.5-8.0$, $\left.P_{c}=0.006\right)$. The women developing psoriasis after 40 years of age $(n=15)$ in turn showed over-expression of the following markers: TNF-308A (66.7\% versus $22 \%$. OR 9.0, 95\% CI: $2.6-$ $\left.31.2, P_{c}=0.004\right)$ and HLA-DR17-DRB1 ${ }^{*} 03-(53 \%$ versus $18 \%$. OR 5.1, 95\% CI: 1.5-12.5, $P_{c}=0.01$ ).

The men developing psoriasis before 40 years of age $(n=$ 45) showed over-expression of the following markers: HLA$\mathrm{C}^{*} 06$ (58\% versus $18.2 \%$. OR 6.2, 95\% CI: $2.5-15.2, P_{c}=$ $0.0001), B^{*} 27$ (42.2\% versus 7.3\%. OR 9.3, 95\% CI: 2.9-30.2, $P_{c}=0.001$ ), and MICA-A9 (60\% versus $27.3 \%$. OR $4.0,95 \%$ CI: 1.7-9.3, $\left.P_{c}=0.001\right)$. The men developing psoriasis after 40 years of age $(n=10)$ in turn showed a significant increase in the following markers: HLA-C 06 (50\% versus $18.2 \%$. OR 4.5, 95\% CI: 1.1-18.5, $P_{c}=0.04$ ), MICB-CA23 (50\% versus 5.5\%. OR 17.3, 95\% CI: 3.2-94.9, $\left.P_{c}=0.001\right)$, C1_4_4 (80\% versus $22 \%$. OR 14.3, 95\% CI: 2.7-76.7, $\left.P_{c}=0.0007\right)$, and MICA-A9 (60\% versus $27.3 \%$. OR 4.0, 95\% CI: $1.0-16.2, P_{c}=0.05$ ) (Tables 3 and 4$)$.

\section{Discussion}

Spondyloarthritic disorders are generally diagnosed more often in men than in women [17]. In reference to the most representative form of these disorders, ankylosing spondylitis, three male cases are documented for every female case [17]. However, since the introduction of the new ASAS criteria for axial spondyloarthritis, these differences are no longer so apparent [18]. PsA appears to be more frequent in men than in women, particularly in its axial presentations [19]. Nevertheless, little is known of the differential clinical expression of PsA between males and females. The explanation for these differences is not clear, and the participation of multifactorial parameters cannot be discarded. In any case, these clinical expression tendencies in men and women with spondyloarthritis appear to persist in the more recently published series [9].

We confirmed the greater peripheral involvement in women with PsA (with more frequent polyarthritis), as well as greater physical functional impairment as measured 
TABLE 3: Distribution of most significant markers in men and women with PsA.

(a)

\begin{tabular}{|c|c|c|}
\hline Marker & PsA females: 55 & Female controls: 55 \\
\hline HLA-C ${ }^{*} 06$ & $\begin{array}{c}31(56.4 \%) \\
\text { OR: } 6.6(2.7-16.1), P_{c}=0.0001\end{array}$ & $9(16.4 \%)$ \\
\hline HLA-C ${ }^{*} 07$ & $\begin{array}{c}27(49 \%) \\
\text { OR: } 2.8(1.3-6.3), P_{c}=0.01\end{array}$ & $14(25.5 \%)$ \\
\hline TNF-308A & $\begin{array}{c}25(45.5 \%) \\
\text { OR: } 3.0(1.3-6.9), P_{c}=0.009\end{array}$ & $12(22 \%)$ \\
\hline MICA-A9 & $\begin{array}{c}33(60 \%) \\
\text { OR: } 3.1(1.4-6.7), P_{c}=0.004\end{array}$ & $18(32.7 \%)$ \\
\hline HLA-B ${ }^{*} 27$ & $\begin{array}{c}15(27.3 \%) \\
\text { OR: } 4.8(1.5-15.5), P_{c}=0.01\end{array}$ & $4(7.3 \%)$ \\
\hline \multicolumn{3}{|c|}{ (b) } \\
\hline Marker & PsA males: 55 & Male controls: 55 \\
\hline HLA-C* 06 & $\begin{array}{c}31(56.4 \%) \\
\text { OR: } 5.8(2.4-13.8), P_{c}=0.0001\end{array}$ & $10(18.2 \%)$ \\
\hline MICA-A9 & $\begin{array}{c}33(60 \%) \\
\text { OR: } 4.0(1.8-8.9), P_{c}=0.001\end{array}$ & $15(27.3 \%)$ \\
\hline HLA-B ${ }^{*} 27$ & $\begin{array}{c}20(36.4 \%) \\
\text { OR: } 7.3(2.3-23.2), P_{c}=0.0004\end{array}$ & $4(7.3 \%)$ \\
\hline
\end{tabular}

TABLE 4: Distribution of most significant markers in men and women with PsA according to age at psoriasis onset (cutoff: 40 years).

(a) PsA females

\begin{tabular}{|c|c|c|c|}
\hline Marker & Age at onset $<40: 40$ & Age at onset $>40: 15$ & Female controls: 55 \\
\hline HLA-C* 06 & $\begin{array}{c}26(65 \%) \\
\text { OR: } 9.5(3.6-24.9), P_{c}=0.0001\end{array}$ & $5(33 \%)$ & $9(16.4 \%)$ \\
\hline HLA-C* 07 & $\begin{array}{c}20(50 \%) \\
\text { OR: } 2.9(1.2-6.9), P_{c}=0.02\end{array}$ & $7(46.7 \%)$ & $14(25.5 \%)$ \\
\hline TNF-308A & $15(37.5 \%)$ & $\begin{array}{c}10(66.7 \%) \\
\text { OR: } 9.0(2.6-31.2), P_{c}=0.0004\end{array}$ & $12(22 \%)$ \\
\hline HLA-DR17 & $4(10 \%)$ & $\begin{array}{c}8(53 \%) \\
\text { OR: } 5.1(1.5-17.5), P_{c}=0.01\end{array}$ & $10(18 \%)$ \\
\hline MICA-A9 & $\begin{array}{c}25(62.5 \%) \\
\text { OR: } 3.4(1.5-8.0), P_{c}=0.006\end{array}$ & $8(53.3 \%)$ & $18(32.7 \%)$ \\
\hline HLA-B ${ }^{*} 27$ & $\begin{array}{c}12(30 \%) \\
\text { OR: } 5.5(1.6-18.5), P_{c}=0.005\end{array}$ & $3(20 \%)$ & $4(7.3 \%)$ \\
\hline \multicolumn{4}{|c|}{ (b) PsA males } \\
\hline Marker & Age at onset $<40: 45$ & Age at onset $>40: 10$ & Male controls: 55 \\
\hline HLA-C ${ }^{*} 06$ & $\begin{array}{c}26(58 \%) \\
\text { OR: } 6.2(2.5-15.2), P_{c}=0.0001\end{array}$ & $\begin{array}{c}5(50 \%) \\
\text { OR: } 4.5(1.1-18.5), P_{c}=0.04\end{array}$ & $10(18.2 \%)$ \\
\hline MICB-CA23 & $1(2.2 \%)$ & $\begin{array}{c}5(50 \%) \\
\text { OR: } 17.3(3.2-94.9), P_{c}=0.001\end{array}$ & $3(5.5 \%)$ \\
\hline C1_4_4 (384) & $12(26.7 \%)$ & $\begin{array}{c}8(80 \%) \\
\text { OR: } 14.3(2.7-76.6), P_{c}=0.0007\end{array}$ & $12(22 \%)$ \\
\hline MICA-A9 & $\begin{array}{c}27(60 \%) \\
\text { OR: } 4(1.7-9.3), P_{c}=0.001\end{array}$ & $\begin{array}{c}6(60 \%) \\
\text { OR: } 4.0(1.0-16.2), P_{c}=0.05\end{array}$ & $15(27.3 \%)$ \\
\hline HLA-B* 27 & $\begin{array}{c}19(42.2 \%) \\
\text { OR: } 9.3(2.9-30.2), P_{c}=0.001\end{array}$ & $1(10 \%)$ & $4(7.3 \%)$ \\
\hline
\end{tabular}


with the HAQ. We do not know whether the same genetic conditioning factors are operative in both males and females with PsA, and the data found in the literature are very limited. Apart from the mentioned relationship between HLA-B27 and the risk of psoriatic spondyloarthritis in males, the only study of note corresponds to that published by Hüffmeier et al., who detected a greater frequency of the R620W mutation of the PTPN22 gene in males with PsA [20].

This study confirmed HLA-C ${ }^{*} 06, \mathrm{~B}^{*} 27$, and MICA-A9 $\left({ }^{*} 002\right)$ as three of the most relevant PsA risk genes within the MHC. All three were significantly overexpressed in men and women with PsA. Our data corroborate the growing importance of the PsA risk genes in the MHC. However, these genetic profiles revealed differential characteristics between men and women. Thus, women with psoriatic nail disease were seen to express the $C^{*} 06$ allele significantly more often than men with the same disease. This finding contrasts with the data published by Gudjonsson et al., who reported greater nail involvement in their HLA-C ${ }^{*} 06$ negative psoriatic patients [21]. These same authors found $\mathrm{C}^{*} 06$ positive women to be younger at the time of onset of psoriasis than $C^{*} 06$ positive men-an observation not recorded in our series [21]. In fact, among $C^{*} 06$ positive men with an age of over 40 years at onset of the disease, onset proved significantly earlier than in $C^{*} 06$ positive women with an age at onset of psoriasis of over 40 years. In turn, the HLA$\mathrm{B}^{*} 27$ positivity was a marker of early onset disease in both sexes confirming recent observations [22].

Patient age at the onset of psoriasis has been a key stratification element in studies of psoriasis. In this sense, it has been known for decades that early psoriasis (manifesting before 40 years of age) is associated with a family history of disease, increased severity, and a stronger correlation to HLA-C ${ }^{*} 06$ positivity, while late psoriasis (manifesting after 40 years of age) appears to be milder, with no clear genetic correlations [23, 24]. Our findings coincide with these observations. However, in our series the impact of the $\mathrm{C}^{*} 06$ allele upon disease risk was found to decrease more clearly with age among females (only $33 \%$ of the women with psoriasis onset above 40 years of age proved positive for this allele) than in men ( $50 \%$ positivity for this allele in individuals with late psoriasis onset). On the other hand, allele 384 of the C4_1_1 microsatellite was associated with late disease in men but not in women-though this allele was in linkage disequilibrium (LD) with $C^{*} 06\left(\lambda_{s}: 0.6\right)$. We found that certain markers that are overexpressed in women, such as C*07, TNF-308A and HLA-DR17 (DRB1* 03), behave differently according to age at the onset of psoriasis. Specifically, $C^{*} 07$ was seen to be elevated in women with early psoriasis, while the latter two markers were significantly overexpressed only in women with late psoriasis. In women there was a certain LD between TNF-308A, and DR17 $\left(\lambda_{\mathrm{s}}\right.$ : $0.3)$. In any case, it is interesting to note that $C^{*} 07$, TNF$308 \mathrm{~A}$, and $\mathrm{DRB1}^{*} 03$ form part of the extended haplotype 8.1, which also contains an allele recently linked to PsA risk (HLA-B ${ }^{*} 08$ ); as a result, it is nowadays difficult to establish which of these alleles are the true disease risk markers [25].

The present work has not been designed as a genetic study, since the small number of patients involved clearly implies a lack of the statistical power needed for such studies. Rather, we aimed to determine whether there are genetic distinctions between men and women that could help explain the little known gender differences in the clinical expression of PsA. Overall, our genetic findings are supported by recent data confirming a good number of our observations referred to the susceptibility role of HLA-B ${ }^{*} 27, C^{*} 06$, and $C^{*} 07$ as risk alleles in PsA [22, 25]. As a novelty, we have seen that the impact of these genetic risk elements differs according to patient's gender and age at the onset of psoriasis.

\section{Conclusions}

In sum, men and women with PsA show differences in the expression of the disease, and this is probably due in part to a differential overexpression of certain MHC genes between the two genders. We consider patient's gender and age at the onset of psoriasis to be key stratification factors that should be considered in future studies of the associations between MHC genes and the risk of PsA.

\section{Acknowledgments}

This work was supported by the Spanish Grants of Fondo de Investigaciones Sanitarias (Institute Carlos III) PI12/01280.

\section{References}

[1] M. Castelino and A. Barton, "Genetic susceptibility factors for psoriatic arthritis," Current Opinion in Rheumatology, vol. 22, no. 2, pp. 152-156, 2010.

[2] J. Martínez-Borra, C. Brautbar, S. González, C. D. Enk, A. López-Vázquez, and C. López-Larrea, "The region of $150 \mathrm{~kb}$ telometic to HLA-C is associated with psoriasis in the Jewish population," Journal of Investigative Dermatology, vol. 125, no. 5, pp. 928-932, 2005.

[3] J. Martínez-Borra, S. González, J. Santos-Juanes et al., "Psoriasis vulgaris and psoriatic arthritis share a $100 \mathrm{~kb}$ susceptibility region telomeric to HLA-C," Rheumatology, vol. 42, no. 9, pp. 1089-1092, 2003.

[4] D. D. Gladman, C. Antoni, P. Mease, D. O. Clegg, and O. Nash, "Psoriatic arthritis: epidemiology, clinical features, course, and outcome," Annals of the Rheumatic Diseases, vol. 64, no. 2, pp. iil4-iil7, 2005.

[5] G. M. Alenius, B. Stenberg, H. Stenlund, M. Lundblad, and S. R. Dahlqvist, "Inflammatory joint manifestations are prevalent in psoriasis: prevalence study of joint and axial involvement in psoriatic patients, and evaluation of a psoriatic and arthritic questionnaire," Journal of Rheumatology, vol. 29, no. 12, pp. 2577-2582, 2002.

[6] S. Gonzalez, J. Martinez-Borra, J. C. Torre-Alonso et al., "The MICA-A9 triplet repeat polymorphism in the transmembrane region confers additional susceptibility to the development of psoriatic arthritis and is independent of the association of Cw*0602 in psoriasis," Arthritis and Rheumatism, vol. 42, no. 5, pp. 1010-1016, 1999.

[7] S. González, J. Martínez-Borra, A. López-Vázquez, S. GarcíaFernández, J. C. Torre-Alonso, and C. López-Larrea, "MICA rather than MICB, TNFA, or HLA-DRB1 is associated with 
susceptibility to psoriatic arthritis," Journal of Rheumatology, vol. 29, no. 5, pp. 973-978, 2002.

[8] S. González, C. Brautbar, J. Martínez-Borra et al., "Polymorphism in MICA rather than HLA-B/C genes is associated with psoriatic arthritis in the Jewish population," Human Immunology, vol. 62, no. 6, pp. 632-638, 2001.

[9] W. Lee, J. D. Reveille, J. C. Davis Jr., T. J. Learch, M. M. Ward, and M. H. Weisman, "Are there gender differences in severity of ankylosing spondylitis? Results from the PSOAS cohort," Annals of the Rheumatic Diseases, vol. 66, no. 5, pp. 633-638, 2007.

[10] L. Eder, A. Thavaneswaran, V. Chandran, and D. D. Gladman, "Gender related differences in severity of psoriatic arthritis," Arthritis and Rheumatism, vol. 63, supplement 10, Article ID 2486C, 2011.

[11] R. Queiro, C. Sarasqueta, J. Belzunegui, C. Gonzalez, M. Figueroa, and J. C. Torre-Alonso, "Psoriatic spondyloarthropathy: a comparative study between HLA-B27 positive and HLAB27 negative disease," Seminars in Arthritis and Rheumatism, vol. 31, no. 6, pp. 413-418, 2002.

[12] R. Queiro, M. Alperi, A. Lopez, C. Sarasqueta, J. L. Riestra, and J. Ballina, "Clinical expression, but not disease outcome, may vary according to age at disease onset in psoriatic spondylitis," Joint Bone Spine, vol. 75, no. 5, pp. 544-547, 2008.

[13] D. D. Gladman, B. Brubacher, D. Buskila, P. Langevitz, and V. T. Farewell, "Psoriatic spondyloarthropathy in men and women: a clinical, radiographic, and HLA study," Clinical and Investigative Medicine, vol. 15, no. 4, pp. 371-375, 1992.

[14] W. Taylor, D. Gladman, P. Helliwell, A. Marchesoni, P. Mease, and H. Mielants, "Classification criteria for psoriatic arthritis: development of new criteria from a large international study," Arthritis and Rheumatism, vol. 54, no. 8, pp. 2665-2673, 2006.

[15] J. M. H. Moll and V. Wright, "Psoriatic arthritis," Seminars in Arthritis and Rheumatism, vol. 3, no. 1, pp. 55-78, 1973.

[16] G. Tamiya, M. Ota, Y. Katsuyama et al., "Twenty-six new polymorphic microsatellite markers around the HLA-B, -C and -E loci in the human MHC class I region," Tissue Antigens, vol. 51, no. 4, part 1, pp. 337-346, 1998.

[17] J. Zochling and E. U. R. Smith, "Seronegative spondyloarthritis," Best Practice and Research: Clinical Rheumatology, vol. 24, no. 6, pp. 747-756, 2010.

[18] M. Rudwaleit, "New approaches to diagnosis and classification of axial and peripheral spondyloarthritis," Current Opinion in Rheumatology, vol. 22, no. 4, pp. 375-380, 2010.

[19] R. Queiro, C. Sarasqueta, J. C. Torre, T. Tinturé, and I. López-Lagunas, "Spectrum of psoriatic spondyloarthropathy in a cohort of 100 Spanish patients," Annals of the Rheumatic Diseases, vol. 61, no. 9, pp. 857-858, 2002.

[20] U. Hüffmeier, A. Reis, M. Steffens et al., "Male restricted genetic association of variant R620W in PTPN22 with psoriatic arthritis," The Journal of investigative dermatology, vol. 126, no. 4, pp. 932-935, 2006.

[21] J. E. Gudjonsson, A. Karason, E. H. Runarsdottir et al., "Distinct clinical differences between HLA-Cw* 0602 positive and negative psoriasis patients - an analysis of 1019 HLA-C- and HLAB-typed patients," Journal of Investigative Dermatology, vol. 126, no. 4, pp. 740-745, 2006.

[22] R. Winchester, G. Minevich, V. Steshenko et al., "HLA associations reveal genetic heterogeneity in psoriatic arthritis and in the psoriasis phenotype," Arthritis and Rheumatism, vol. 64, no. 4, pp. 1134-1144, 2011.
[23] T. Henseler and E. Christophers, "Psoriasis of early and late onset: characterization of two types of psoriasis vulgaris," Journal of the American Academy of Dermatology, vol. 13, no. 3, pp. 450-456, 1985.

[24] R. Queiro, S. Alonso, M. Alperi, M. Fernández, and C. Sarasqueta, "Stratification by age of onset with 30 years as age limit is an effective means of identifying PSORS1-associated psoriasis in patients with psoriatic arthritis," Joint Bone Spine, vol. 78, no. 6, pp. 581-583, 2011.

[25] L. Eder, V. Chandran, F. Pellet et al., "Human leucocyte antigen risk alleles for psoriatic arthritis among patients with psoriasis," Annals of the Rheumatic Diseases, vol. 71, no. 1, pp. 50-55, 2012. 


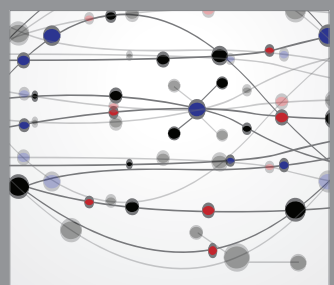

The Scientific World Journal
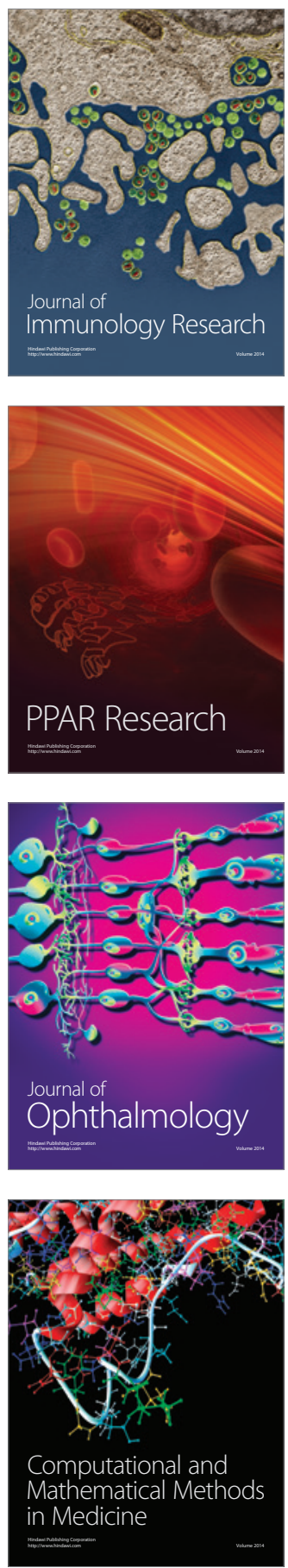

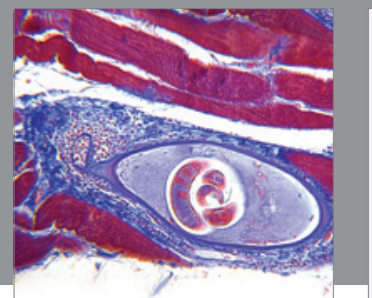

Gastroenterology

Research and Practice
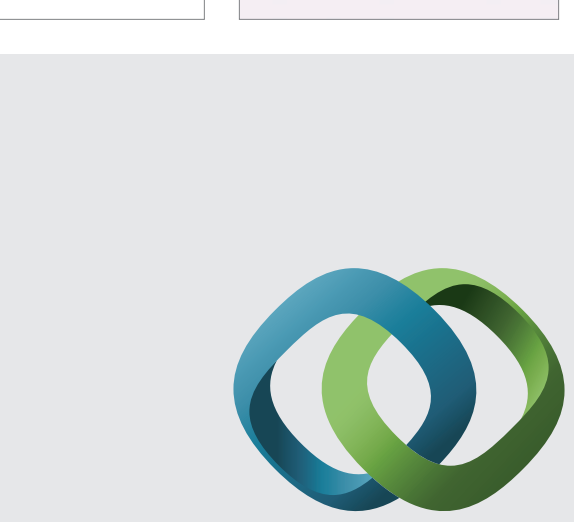

\section{Hindawi}

Submit your manuscripts at

http://www.hindawi.com
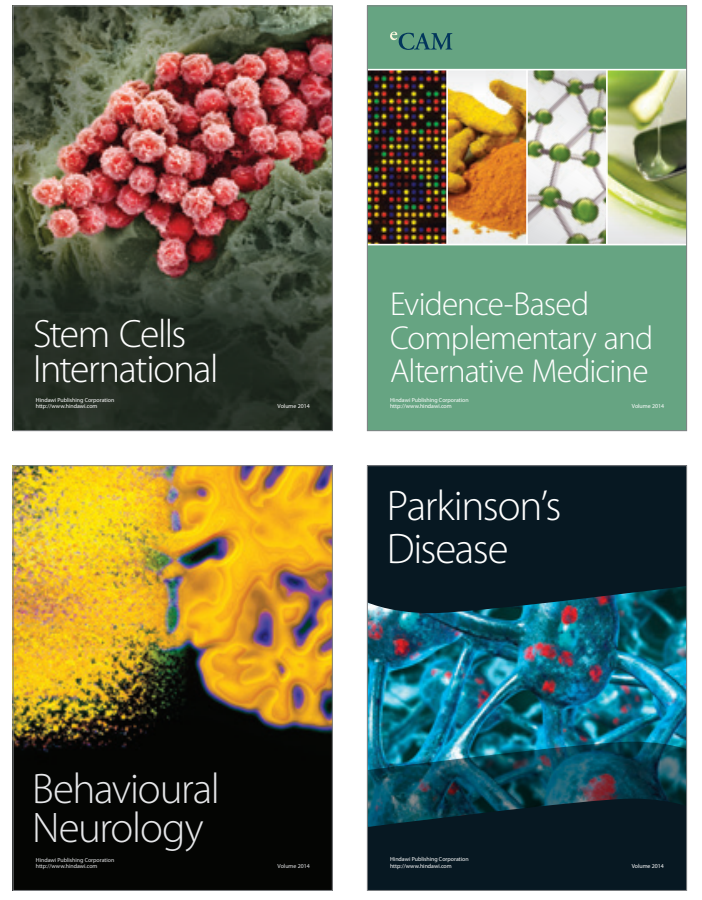
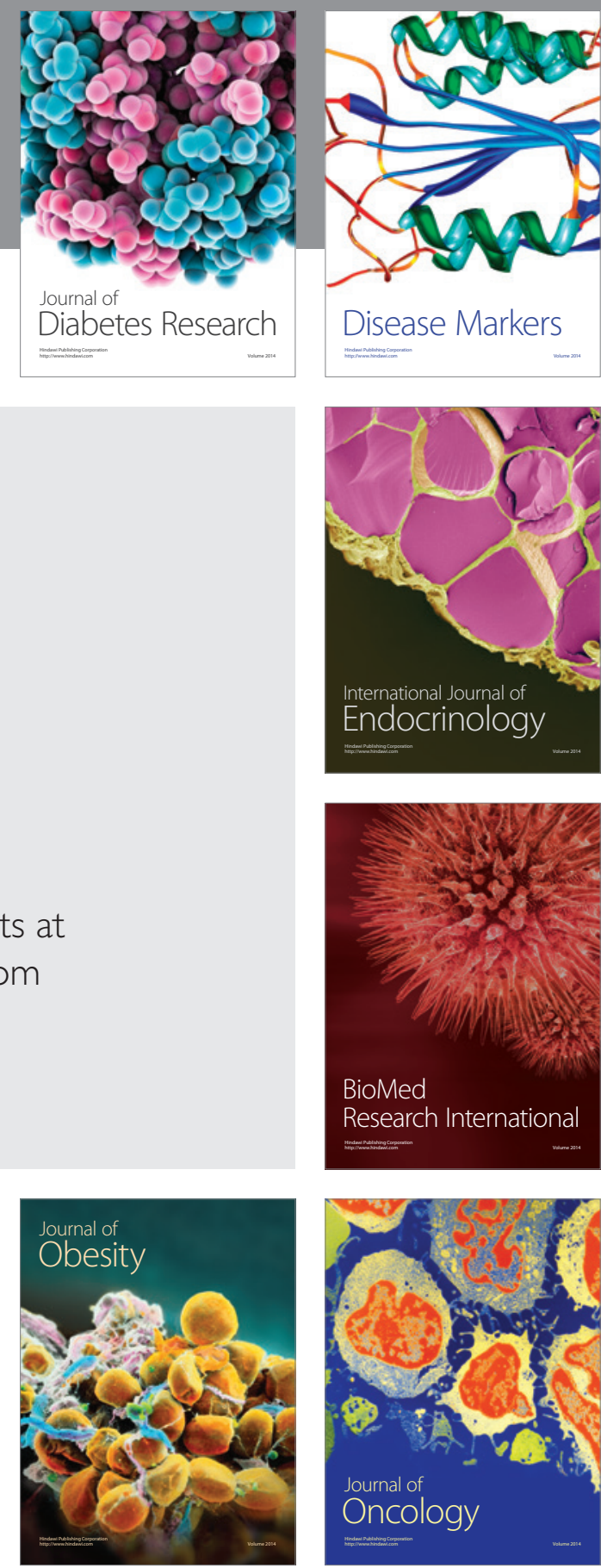

Disease Markers
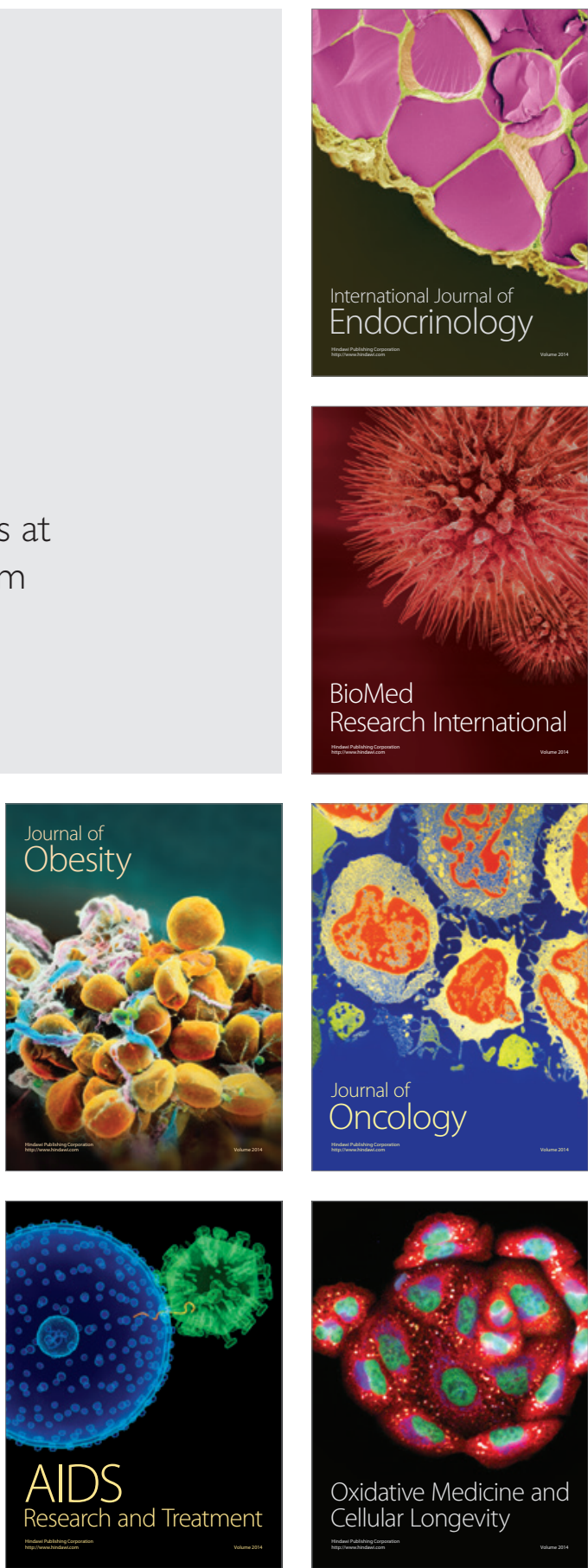